\title{
Molecular Characterization of Salmonella Serovars Anatum and Ealing Associated with Two Historical Outbreaks, Linked to Contaminated Powdered Infant Formula
}

OPEN ACCESS

Edited by:

Julio Parra-Flores,

University of the Bío Bío, Chile

Reviewed by:

Ariadnna Cruz-Córdova,

Hospital Infantil de México Federico

Gómez, Mexico

Hongxia Wang,

University of Alabama at Birmingham,

USA

${ }^{*}$ Correspondence:

Séamus Fanning

sfanning@ucd.ie

${ }^{\dagger}$ These authors have contributed equally to this work

Specialty section

This article was submitted to

Food Microbiology,

a section of the journal

Frontiers in Microbiology

Received: 15 August 2016 Accepted: 05 October 2016 Published: 21 October 2016

Citation:

Gunn L, Finn S, Hurley D, Bai L.

Wall E, Iversen C, Threlfall JE and

Fanning S (2016) Molecular

Characterization of Salmonella

Serovars Anatum and Ealing

Associated with Two Historical

Outbreaks, Linked to Contaminated

Powdered Infant Formula.

Front. Microbiol. 7:1664.

doi: 10.3389/fmicb.2016.01664

\section{Lynda Gunn ${ }^{1+}$, Sarah Finn ${ }^{1+}$, Daniel Hurley ${ }^{1}$, Li Bai ${ }^{1,2}$, Ellen Wall ${ }^{1}$, Carol Iversen ${ }^{1}$, John E. Threlfall ${ }^{3}$ and Séamus Fanning ${ }^{1,2,4 *}$}

${ }^{1}$ UCD-Centre for Food Safety, School of Public Health, Physiotherapy and Sports Science, University College Dublin, Dublin, Ireland, ${ }^{2}$ Key Laboratory of Food Safety Risk Assessment, Ministry of Health, China National Center for Food Safety Risk Assessment, Beijing, China, ${ }^{3}$ Health Protection Agency, London, UK, ${ }^{4}$ Institute for Global Food Security, Queen's University Belfast, Belfast, UK

Powdered infant formula (PIF) is not intended to be produced as a sterile product unless explicitly stated and on occasion may become contaminated during production with pathogens such as Salmonella enterica. This retrospective study focused on two historically reported salmonellosis outbreaks associated with PIF from the United Kingdom and France, in 1985 and 1996/1997. In this paper, the molecular characterization of the two outbreaks associated Salmonella serovars Anatum and Ealing is reported. Initially the isolates were analyzed using pulsed-field gel electrophoresis (PFGE), which revealed the clonal nature of the two outbreaks. Following from this two representative isolates, one from each serovar was selected for whole genome sequencing (WGS), wherein analysis focused on the Salmonella pathogenicity islands. Furthermore, the ability of these isolates to survive the host intercellular environment was determined using an ex vivo gentamicin protection assay. Results suggest a high level of genetic diversity that may have contributed to survival and virulence of isolates from these outbreaks.

Keywords: PFGE, whole genome sequencing, Salmonella pathogenicity islands, core genome, infection model

\section{INTRODUCTION}

Infant foods, such as baby cereals and powdered infant milk formulae (PIF), can act as vectors for pathogenic microorganisms of importance to human health as technology is currently unavailable to manufacture these foods as sterile products. Thus, despite the implementation of good manufacturing practices PIF may, on occasion, become contaminated with pathogens during production. Those pathogens that present the greatest threat to infant health include Cronobacter species (formerly known as Enterobacter sakazakii) and Salmonella enterica, two bacterial genera designated by the World Health Organisation (WHO) as Class A pathogens. Following the consumption and ingestion of contaminated foods, clinical signs of infection include gastroenteritis which can progress to bacteraemia and meningitis (Cahill et al., 2008). Numerous Salmonella outbreaks associated with contaminated infant foods have been documented since the 1950s and 1960s (Brouard et al., 2007). 
In this study two Salmonella enterica serovars implicated in PIF outbreaks were investigated. The first outbreak occurred in 1985, when Salmonella Ealing, was identified and linked to cases of salmonellosis in the United Kingdom. Some 70 individuals were affected, the majority of these being infants. A subsequent investigation showed low numbers of Salmonella organisms in the PIF ( 1.6 CFU/450 g) making their detection challenging during routine testing of the food product (Rowe et al., 1987). A second outbreak was reported between 1996/1997 and this was associated with PIF contaminated with $S$. Anatum. On this occasion, the outbreak centered in the United Kingdom and France (Threlfall et al., 1998). Public Health England reported an increase in $S$. Anatum isolations from children less than 1 year old and due to the young age of these patients infant food was suspected to have been responsible for the transmission of the aetiological agent.

This study reports the molecular characterization of Salmonella enterica serovars Anatum and Ealing, which were connected to the referenced outbreaks through contaminated PIF. Isolates were initially characterized by macrorestrictionbased DNA fingerprinting and later further analyzed by whole genome sequencing (WGS), to observe the genetic diversity of these outbreaks and compare them to other Salmonella serovars. Infection assays were performed for two selected isolates from each of these outbreaks. The subsequent analysis focused on Salmonella pathogenicity Island (SPI) comparison as well as bacterial survival in ex-vivo infection models, to observe potential associations between survival and genetic diversity.

\section{MATERIALS AND METHODS}

\section{Bacterial Culture}

Thirty seven bacterial isolates were included in this study comprising of $12 \mathrm{~S}$. Anatum and $25 \mathrm{~S}$. Ealing. All were stored on beads at $-80^{\circ} \mathrm{C}$ and sub-cultured on tryptone soya agar (TSA) plates at $37^{\circ} \mathrm{C}$ when required.

\section{Molecular Macrorestriction Digest by Xba1 and Spe1}

PFGE pulsotypes were obtained for all isolates following digestion with $X b a 1$ restriction enzyme as previously described by Ribot et al. (2006). Isolates that produced indistinguishable patterns with this enzyme were subsequently reanalyzed using a second enzyme, SpeI as described by Zheng et al. (2007). Visualization of DNA profiles was carried out using Gel Logic 1500 imaging system. The TIFF files were imported in BioNumerics v.5.1 and dendrograms constructed using the UPGMA and DICE algorithms with 1.0\% optimization and 1.5\% tolerance.

\section{Whole Genome Sequencing and Comparative Phylogenetic Analysis of Core Salmonella Genomes}

Whole genome sequencing of isolates was performed using the Illumina MiSeq platform. Library preparation was performed using Nextera XT kit (v3 chemistry) according to manufacturer's instructions producing 300 bp paired end reads. Subsequent raw sequence data was assessed using FastQC. The reads were error corrected using the BFC algorithm before a relaxed quality trim using a sliding window as implemented in Trimmomatic v0.33 (Bolger et al., 2014; Li, 2015). De novo genome assemblies were produced using SPAdes assembler v3.6.2 using the default $k$-mer size selection for $300 \mathrm{bp}$ reads with careful mode enabled (used for mismatch error, indel and error correction; Bankevich et al., 2012).

For core genome analysis, comparisons were selected by online BLAST similarity searches of the largest contiguous sequence from S. Anatum CFS0056 and S. Ealing CFS0080 assemblies. Other serovars were included as they have been associated with food-borne outbreaks previously. Protein sequences from all strains identified by annotation with Prokka (version 1.11) were clustered using the pan-genome pipeline Roary (version 3.6.2) (Seemann, 2014; Page et al., 2015). Visualization of the pan-genome was carried out using Anvi'o (version 2.0.2) (Eren et al., 2015).

\section{Comparative Analysis of Selected Salmonella Pathogenicity Islands (SPI)}

The differences in SPI associated genes from SPI-1 through -5 were investigated as previously described using standalone BLAST + v2.4 and comparing these against the corresponding loci from $S$. Typhimurium ST4/74 as the reference genome ${ }^{1}$. The resulting nucleic acid sequences were converted to amino acid sequences using Biopython (version 1.66) and the percentage similarity of the SPI proteins from $S$. Anatum and $S$. Ealing when compared against the reference were determined using the Needleman-Wunsch alignment with default settings through the EMBOSS analysis software (version 6.6.0) (Needleman and Wunsch, 1970). The raw sequencing data and de novo assemblies have been deposited at the Sequence Read Archive/GenBank for S. Anatum CFS0056 (SRP081283/SAMN05560363) and S. Ealing CFS0080 (SRP081283/SAMN05560364).

\section{Ex vivo Gentamicin Protection Assay}

A gentamicin protection assay was used to observe whether the isolates could survive phagocytosis using $S$. Typhimurium ST4/74 as the reference strain adapted from protocols as described previously (Lathrop et al., 2015). Briefly THP-1 monocytes were grown in antibiotic-free RPMI 1640 media (Sigma-Aldrich) supplemented with $10 \%[\mathrm{v} / \mathrm{v}]$ heat inactivated FBS and incubated at $37^{\circ} \mathrm{C}$ with $5 \% \mathrm{CO}_{2}$. Cells were detached with $1 \%$ Triton X-100 and seeded at a density of $1 \times 10^{5}$ cells $/ \mathrm{mL}$ per well in 24 well-plates. Monocytes were differentiated to adherent macrophages by supplementing media with $20 \mathrm{ng} / \mathrm{mL}$ phorbol 12-myristate 13-acetate (PMA) for 5 days.

Prior to infection, bacterial isolates were diluted in complete media to $1 \times 10^{6}$ cells $/ \mathrm{mL}$ for a MOI of 10:1 and incubated at $37^{\circ} \mathrm{C}$ for $1 \mathrm{~h}$. Macrophages were washed with $1 \mathrm{~mL}$ Hank's Balanced Salt Solution (HBSS), three times, before $1 \mathrm{~mL}$ of the bacterial suspension, was added to each well; $1 \mathrm{~mL}$ of complete media was added to control wells. The plates were centrifuged

${ }^{1}$ danieljhurley/atypical-Salmonella. GitHub Available online at: https://github. com/danieljhurley/atypical-Salmonella (Accessed August 10, 2016). 


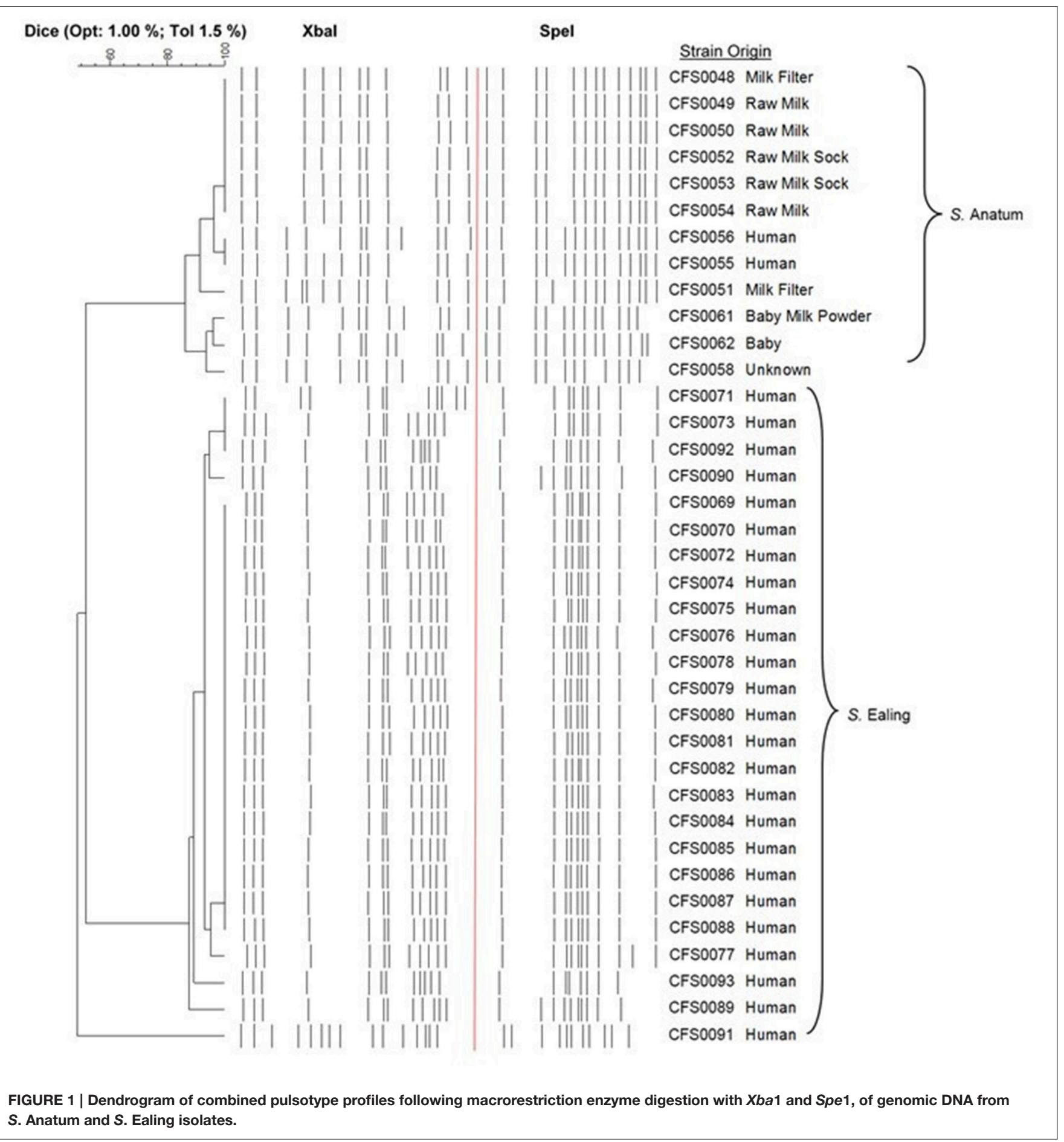

at $300 \times \mathrm{g}$ for $5 \mathrm{~min}$ and incubated for $1 \mathrm{~h}$ to allow for phagocytosis. Following phagocytosis, the cells were washed with $1 \mathrm{~mL}$ HBSS three times. To kill exposed bacteria $1 \mathrm{~mL}$ complete media supplemented with $100 \mu \mathrm{g} / \mathrm{mL}$ gentamicin was added to each well before incubation for $1 \mathrm{~h}$. After the wells were washed with $1 \mathrm{~mL}$ HBSS. A final $1 \mathrm{~mL}$ of complete media supplemented with $20 \mu \mathrm{g} / \mathrm{mL}$ gentamicin was then added to each well before incubation at for the desired time points. At each time point cells were washed three times with $1 \mathrm{~mL}$ HBSS, after $1 \mathrm{~mL} 1 \%$ [v/v] Triton X-100 Phosphate Buffered Saline (PBS) solution was added to the cells and incubated at room temperature for $10 \mathrm{~min}$. The resulting supernatants were decimally diluted in PBS, $100 \mu \mathrm{L}$ aliquots of the dilutions were plated onto LB agar and incubated for $18 \mathrm{~h}$ at $37^{\circ} \mathrm{C}$ before enumeration. 


\section{RESULTS}

\section{Molecular Macrorestriction Digest by Xba1 and Spe1}

The pulsotypes of all 37 isolates were investigated to assess the genetic relationship(s) between those Salmonella isolates linked with the two outbreaks in this study. A dendrogram based on the combined pulsotypes from Xba1 and Spe1 digest representing serovars Anatum and Ealing, is shown in Figure 1. In the case of $S$. Ealing, the pulsotype of the isolate denoted as CFS0091 a non-outbreak strain was notably different ( $\leq 7$ band difference; when digested with $X b a 1$ or Spe1) when compared to the other pulsotypes obtained for other members of this serovar (estimated at $\sim 50 \%$ similarity). The majority of $S$. Ealing from the outbreak have very similar pulsotypes with the exception of isolates CFS0089, -0093, -0077, -0071, -0073, -0092, and -0090 which displayed more diverse profile (1-3 band differences).

For S. Anatum isolates CFS0048-0050 and CFS0052-0056 originally cultured from equipment and clinical cases, these produced very similar pulsotypes, displaying a high degree of similarity (>95\%), suggesting a potential epidemiological link. Other isolates from the same serovar showed differences in their restriction patterns (2-4 band difference) with less similarity to clinical isolates ( $85-90 \%$ similarity) making a link less probable.

\section{Determination of the Whole Genome Sequences for Salmonella Anatum CFS0056 and Ealing CFS0080}

Due to the indistinguishable nature of these outbreaks based on their pulsotypes as shown in the PFGE dendrogram (Figure 1), two representative isolates from these PIF outbreaks, $S$. Anatum CFS0056, and S. Ealing CFS0080 were selected, as both were cultured from clinical sources. The whole genome sequences of $S$. Anatum and S. Ealing were obtained using the MiSeq platform. Using these data, comparison of the genomes could provide additional insights in to these historical bacterial isolates, the basic statistics of the genome assemblies from this study are shown in Table 1; S. Anatum CFS0056 and S. Ealing CFS0080 displayed the typical Salmonella DNA characteristics.

Availing of the Roary pipeline and Anvi'o visualization tool for broad genomic comparison, the core and accessory genomes for both serovars can be determined and then compared to a series of Salmonella isolates as shown in Figure 2. Salmonella Anatum CFS0056 and $S$. Ealing shared $\sim 43 \%$ of all unique protein clusters identified (numbering 3544 of 8255) when compared against other selected isolates of this bacterial genus (Figure 2). A phylogenetic analysis using a core genome alignment of various Salmonella serovars showed that S. Ealing CFS0080 and S. Agona SL483 displayed a high degree of genetic similarity. Salmonella Agona SL483 was isolated in 2008 and epidemiologically linked to cases of food-borne human infections associated with the consumption of contaminated dried cereal foods (Fricke et al., 2011). In contrast S. Anatum CFS0056 from this study shared a high degree of similarity with other Anatum serovar isolates, although 260 protein clusters were
TABLE 1 | Comparitive features of the genomes of $S$. Anatum and S. Ealing with S. Typhimurium ST4/74.

\begin{tabular}{lccc}
\hline $\begin{array}{l}\text { Features/Salmonella } \\
\text { serovars }\end{array}$ & $\begin{array}{c}\text { Typhimurium } \\
\text { ST4/74 }\end{array}$ & $\begin{array}{c}\text { Anatum } \\
\text { CFS0056 }\end{array}$ & $\begin{array}{c}\text { Ealing } \\
\text { CFS0080 }\end{array}$ \\
\hline Accession number & NC_016857 & - & - \\
SRA number & n/a & SRP081283 & SRP081283 \\
Assembly contigs & $\mathrm{n} / \mathrm{a}$ & 486,442 & 51 \\
Assembly N50 & 4.87 & 4.87 & 408,530 \\
Genome size (Mbp) & 52.18 & 52.42 & 4.82 \\
\%GC & 4782 & 4563 & 52.17 \\
Predicted coding & & & 4471 \\
sequences (CDS) & 22 & 9 & 10 \\
No. of rRNA operons & 85 & 78 & 83 \\
No. of tRNA operons & 1 & 1 & 1 \\
No. of tmRNA operons & & & \\
\hline
\end{tabular}

unique to $S$. Anatum CFS0056 among the isolates included in this analysis. Overall, $S$. Ealing and $S$. Anatum shared seven unique proteins in comparison to the other isolates included in the analysis including TU1 elongation factor 1 along with hypothetical proteins.

\section{Comparative Analysis of Selected Salmonella Pathogenicity Islands (SPI) from the Genomes of Serovars Anatum and Ealing}

SPIs are horizontally acquired genetic cassettes that play a major role in Salmonella survival and virulence. The SPI-containing proteins from S. Anatum CFS0056 and S. Ealing CFS0080 appear to share high levels of similarity (ranging from $<95$ to $100 \%$ ) when compared against the reference $S$. Typhimurium ST4/74 (Figure 3). Proteins from SPI-1, -2, -4, and -5 display varying degrees of diversity, in comparison to SPI-3 which appears to be highly conserved. Upon closer inspection, S. Ealing CFS0080 demonstrated an overall lower sequence similarity in comparison to that observed for $S$. Anatum CFS0056 from this study. A total of 11 proteins were not detected from this analysis in one or both strains, a feature that should be interpreted with caution.

\section{Intracellular Survival of S. enterica Serovars in Human Macrophages}

To study the ability of these two isolates to survive phagocytosis, ex vivo infections were performed using differentiated human THP-1 macrophages in a gentamicin protection assay. Salmonella Typhimurium ST4/74 was included as a reference strain. Infections were carried out at a Multiplicity $\underline{\text { Of Infection (MOI) }}$ of 10:1. Viable internalized bacteria were enumerated at $2,4,8$, and 24 Hours Post Infection (HPI). Of the two serovars Anatum CFS0056 and Ealing CFS0080 tested in this study, all were found to persist within THP-1 macrophages for a period of up to 24 HPI. Although, the number of $S$. Ealing colonies recovered at 24 HPI was lower in comparison to $S$. Anatum and $S$. Typhimurium, pairwise comparison using ANOVA indicates this difference was statistically significant $(p=0.02)$. 


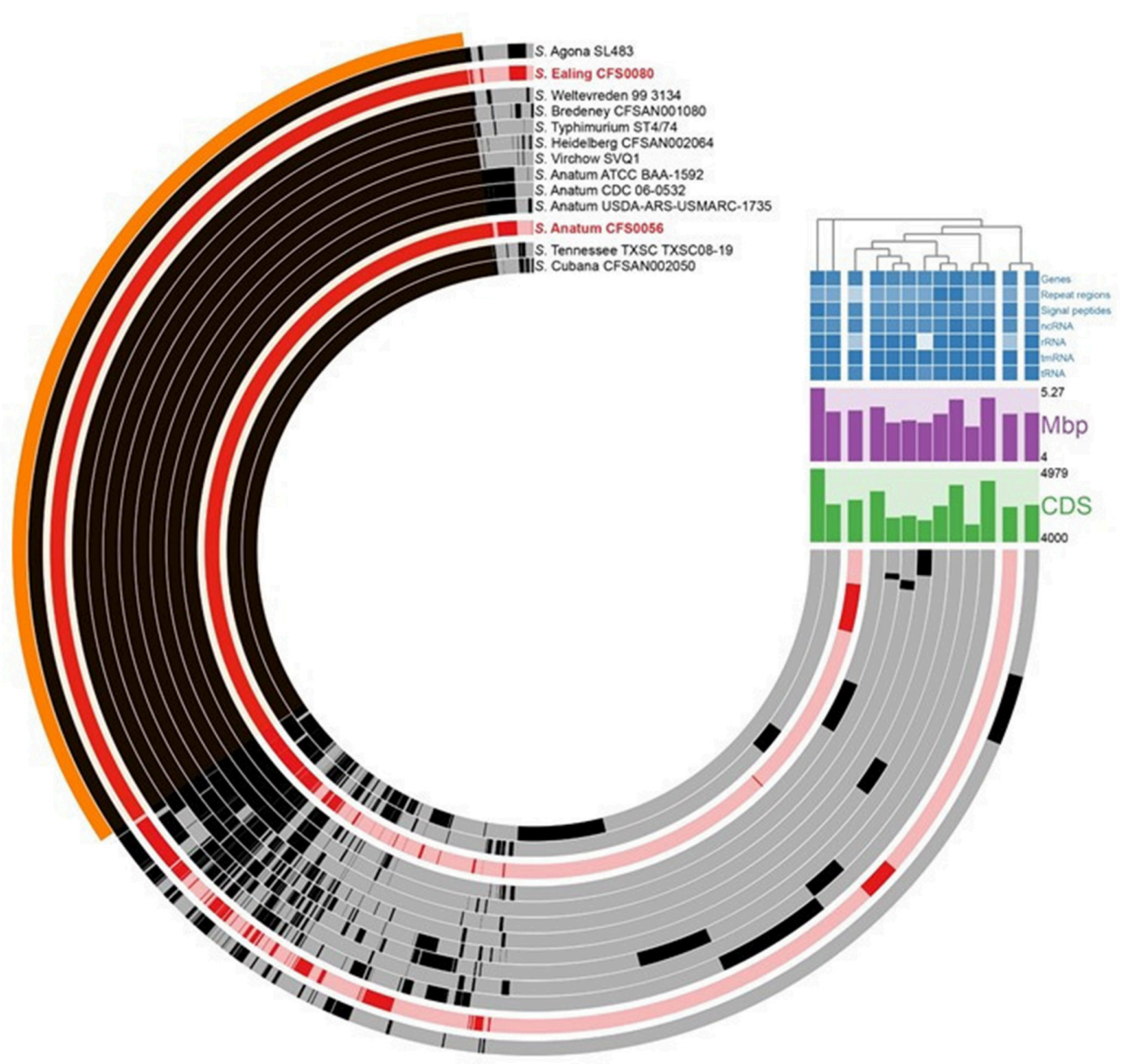

FIGURE 2 | Comparitive Anvi'o phylogenetic analysis of core genome from selected Salmonella serovars compared against S. Anatum CFS0056 and S. Ealing CFS0080. Regions of the circular map shown in black denote similar content between isolates. Red colored regions shown in the circular map denote the genomes of the S. Anatum CFS0056 and S. Ealing CFS0080 study isolates. The region of the map marked in orange represents the core genome across all isolates. The coding sequence content (denoted as CDS; Green); the genome sizes (denoted as Mbp; Purple, see also Table 1) and a cluster diagram are also shown.

\section{DISCUSSION}

PIF can become contaminated with bacteria of importance to human health, some of which may be pathogenic (including Salmonella species among others; Mullane et al., 2007). It is a constant challenge for the PIF industry generally, to be able to detect Class A pathogens such as Salmonella species in this food matrix prior to distribution due to the low numbers that are present on occasion. Failure to do so, can lead to salmonellosis infection among infants, some of which may be life-threatening (Cahill et al., 2008). Similarly, failures in the food safety management of these associated food production facilities can result in the environment and final product becoming contaminated. This retrospective study investigated Salmonella serovars Anatum and Ealing cultured from two historical outbreaks and compared their molecular characteristics.

Molecular fingerprinting identified two clonal clusters consistent with the two serovars, Anatum and Ealing. Overall both serovars displayed a high degree of genetic diversity with profiles sharing similarities ranging from 85 to $100 \%$. The majority of $S$. Ealing produced pulsotypes that were indistinguishable using Xba1 or Spe1 alone (Figure 1), with the exception of isolate CFS0091, which was determined to be un-related to the outbreak. Comparative analysis of the pulsotypes obtained for S. Anatum isolates CFS0048-0050 and CFS0052-0056 (Figure 1), suggested that these isolates of clinical origin and from the PIF production environment were linked during the 1996/1997 outbreak. Whole genome sequencing was performed on S. Anatum CFS0056 and S. Ealing CFS0080. These isolates were selected as representatives due to their apparent clonal nature and clinical isolation.

Analysis of the core genome revealed S. Anatum CFS0056 and $S$. Ealing CFS0080 share $\sim 43 \%$ of the unique protein clusters identified by Prokka annotation. In this study $S$. Anatum was found to be more homologous when compared with other representatives of the same serovar, though with some unique regions being identified (Figure 2). These unique genomic loci could be related to the survival phenotype of this isolate in 


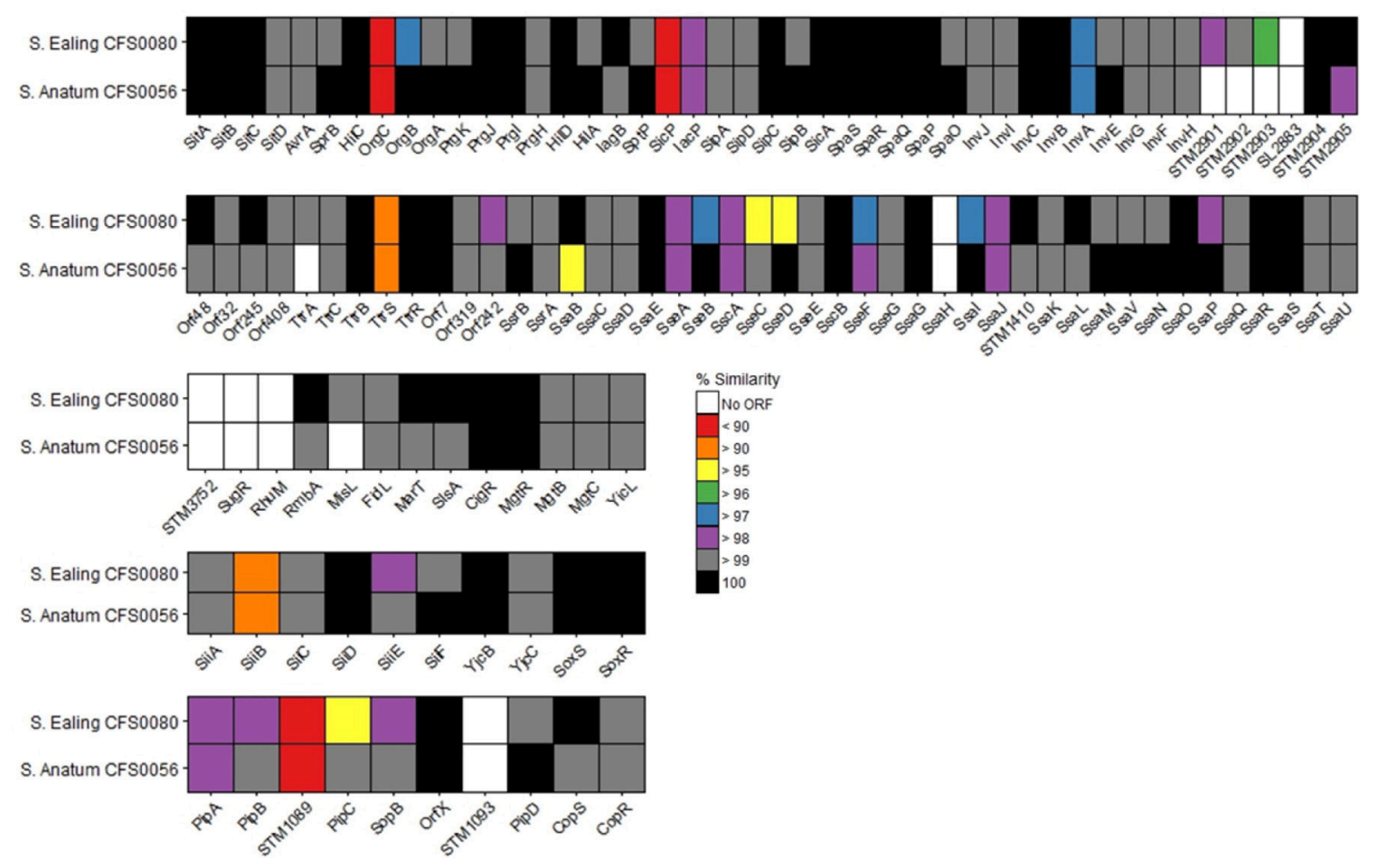

FIGURE 3 | Percentage similarity of SPI proteins 1-5 detected within S. Anatum CFS0056 and S. Ealing CFS0080 compared against reference strain S. Typhimurium ST4/74.

the PIF production environment and therefore require further exploration. Moreover, $S$. Anatum is not typically associated with milk-related outbreaks but is more commonly encountered in meat-processing settings (Ebuchi et al., 2006; Marasini et al., 2016). In the analysis displayed here $S$. Anatum also demonstrated a distant relationship to $S$. Typhimurium together with the Salmonella serovars Virchow and Heidelberg. Both of these latter isolates were previously cultured from outbreaks involving vegetable and meat related products. When analyzed, Salmonella Ealing was found to cluster only with S. Agona SL483 a pathogen also implicated in a food-related outbreak linked dried cereal food product, suggesting the two isolates have similar genetic elements which could contribute to survival in low moisture environments.

Comparative analysis at the level of the amino acid sequence of SPI-1 through to SPI-5 was performed as these are the most common SPIs in all Salmonella enterica serovars sequenced to date. These SPIs code for virulence factors which facilitate internalization of the pathogen and have an impact on the survival of the bacterium in the host intercellular environment. Of specific interest were SPI-1 and -2 that encode genes that function in bacterial virulence including adhesion, and vacuole stabilization. Figure 3 presents the comparison in the form of a heat map that shows a near complete complement of genes was detected across all isolates for SPI-1-SPI-5 together with a high level of amino acid similarity being observed between the outbreak and reference strains. This comparative

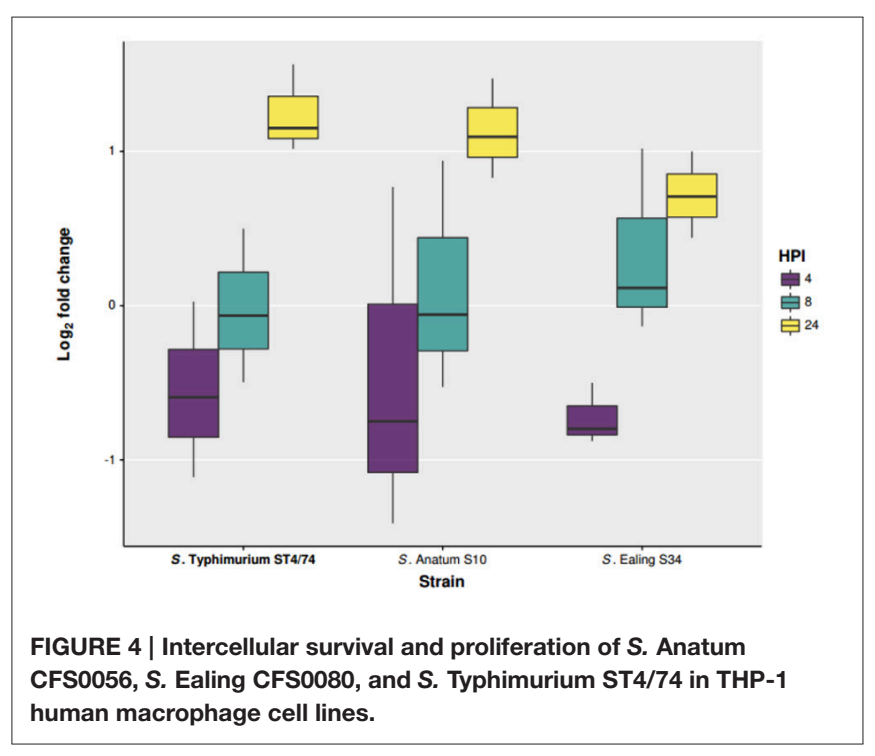

approach also identified proteins that are more diverse when compared to the reference, including those with key roles in intercellular survival and virulence (reviewed in McGhie et al., 2009; Fricke et al., 2011; Ruby et al., 2012; Lathrop et al., 2015). Proteins located within SPI-1, -2, -4, and -5 showed the greatest diversity and these in particular included 
proteins critical in infection and persistence such as SiiEFG that functions in bacterial adhesion and invasion, SseFG, SopB, which are essential in Salmonella containing vacuole stabilization and replication and PipABC which are involved in entropathogenicity (Wood et al., 1998; Morgan et al., 2007; Ibarra and Steele-Mortimer, 2009). SPI-3 showed a high degree of similarity with the reference strain. Of note, three proteins (RhuM, SugR, STM3752), were not detected in this analysis, an observation that does not necessarily mean these genes were absent.

Overall S. Ealing CFS0080 displayed a greater degree of sequence diversity across the SPI island proteins in comparison to $S$. Anatum CFS0056, when compared to S. Typhimirium ST4/74. The consequences of sequence divergence within the SPI genes is not clear at present but targeted knockout studies of selected genes across SPIs 1-5 suggest that if pseudogenes accumulate in the bacterial genome, an impaired virulence phenotype could result (Rychlik et al., 2009). This particularly relates to SPI-1 and SPI-2 which are essential for host adaption and systemic infection. If these two SPIs become non-functional the virulence and survival of the strains, in the human host could be impaired at the level of the phenotype (Ibarra and Steele-Mortimer, 2009).

Both S. Anatum CFS0056 and S. Ealing CFS0080 survived for $24 \mathrm{HPI}$ in human THP-1 macrophages (Figure 4). The numbers of bacteria recovered after 4 and 8 HPI were comparable to those of S. Typhimurium ST4/74. However, after $24 \mathrm{HPI}$, the cell count for $S$. Ealing CFS0080 was found to be less than that of S. Anatum CFS0056 and S. Typhimurium ST4/74. The adaption of Salmonella to the stresses experienced in the host-macrophage environment has been associated with the development of viable non-replicating cells (Helaine et al., 2010, 2014). Further, another report has also observed the potential link between reduced virulence and increased SPI variability using cell infection models (McWhorter and Chousalkar, 2015). The variation in sequence conservation across SPI proteins could be

\section{REFERENCES}

Bankevich, A., Nurk, S., Antipov, D., Gurevich, A. A., Dvorkin, M., Kulikov, A. S., et al. (2012). SPAdes: a new genome assembly algorithm and its applications to single-cell sequencing. J. Comput. Biol. 19, 455-477. doi: $10.1089 / \mathrm{cmb} .2012 .0021$

Bolger, A. M., Lohse, M., and Usadel, B. (2014). Trimmomatic: a flexible trimmer for Illumina sequence data. Bioinformatics 30, 2114-2120. doi: 10.1093/ bioinformatics/btu170

Brouard, C., Espié, E., Weill, F. X., Kérouanton, A., Brisabois, A., Forgue, A. M., et al. (2007). Two consecutive large outbreaks of Salmonella enterica serotype Agona infections in infants linked to the consumption of powdered infant formula. Pediatr. Infect. J. 26, 148-152. doi: 10.1097/ 01.inf.0000253219.06258.23

Cahill, S. M., Wachsmuth, I. K., Costarrica, M. de L. and Embarek, P. K. B. (2008). Powdered infant formula as a source of Salmonella infection in infants. Clin. Infect. Dis. 46, 268-273. doi: 10.1086/ 524737

Ebuchi, S., Baba, A., Uryu, K., and Hiwaki, H. (2006). Two outbreaks caused by Salmonella Derby and $S$. Anatum at grilled-meat restaurants in Fukuoka city. Jpn. J. Infect. Dis. 59, 405-406. associated with non-functioning proteins leading to attenuated in-vivo survival.

\section{CONCLUSION}

In this study, the molecular characteristics of $S$. Anatum and $S$. Ealing cultured from powdered infant formula related outbreaks were studied. Pulsotyping with PFGE demonstrated the clonal nature of the outbreak strains and this feature facilitated their distinction from unrelated isolates of the same serovar. WGS and subsequent analysis of selected representatives of these two serovars identified genomic regions that were common, but also showed that a large accessory genome could be identified. Macrophage infection studies confirmed the ability of both serovars to persist in the host cell for similar periods but at different bacterial cell numbers, reflecting differences in sequence conservation among the SPI islands studied.

Overall the results from this study provide a useful foundation to begin to extend the investigation of how these unique bacterial isolates adapted to the PIF-processing environment, a necessary prelude to the outbreaks reported in each case.

\section{AUTHOR CONTRIBUTIONS}

LG-drafted the manuscript (MS), collated, and reviewed all results and contributed to the WGS and subsequent bioinformatics analysis. SFi-assembled the collection of isolates and performed the initial geno- and phenotypic-studies whilst contributing to the drafting of the MS. DH-carried out cell culture work; evaluated all of the WGS data and carried to the bioinformatics analysis. LB-performed cell culture work. EW-performed phenotype characterization of the isolates. CIassembled the collection of isolates and performed the initial geno- and phenotypic-studies whilst contributing to the drafting of the MS. JT-provided bacteria and background information. $\mathrm{SFa}-$ co-ordinated the study.

Eren, A. M., Esen, Ö. C., Quince, C., Vineis, J. H., Morrison, H. G., Sogin, M. L., et al. (2015). Anvi'o: an advanced analysis and visualization platform for 'omics data. PeerJ 3:e1319. doi: 10.7717/peerj.1319

Fricke, W. F., Mammel, M. K., McDermott, P. F., Tartera, C., White, D. G., Leclerc, J. E., et al. (2011). Comparative genomics of 28 Salmonella enterica isolates: evidence for CRISPR-mediated adaptive sublineage evolution. J. Bacteriol. 193, 3556-3568. doi: 10.1128/JB.00297-11

Helaine, S., Cheverton, A. M., Watson, K. G., Faure, L. M., Matthews, S. A., and Holden, D. W. (2014). Internalization of Salmonella by macrophages induces formation of nonreplicating persisters. Science 343, 204-208. doi: 10.1126/science. 1244705

Helaine, S., Thompson, J. A., Watson, K. G., Liu, M., Boyle, C., and Holden, D. W. (2010). Dynamics of intracellular bacterial replication at the single cell level. Proc. Natl. Acad. Sci. U.S.A. 107, 3746-3751. doi: 10.1073/pnas.1000041107

Ibarra, J. A., and Steele-Mortimer, O. (2009). Salmonella - the ultimate insider. Salmonella virulence factors that modulate intracellular survival. Cell. Microbiol. 11, 1579-1586. doi: 10.1111/j.1462-5822.2009.01368.x

Lathrop, S. K., Binder, K. A., Starr, T., Cooper, K. G., Chong, A., Carmody, A. B., et al. (2015). Replication of Salmonella enterica serovar typhimurium in human monocyte-derived macrophages. Infect. Immun. 83, 2661-2671. doi: 10.1128/IAI.00033-15 
Li, H. (2015). BFC: correcting Illumina sequencing errors. Bioinformatics 31, 2885-2887. doi: 10.1093/bioinformatics/btv290

Marasini, D., Abo-Shama, U. H., and Fakhr, M. K. (2016). Complete genome sequences of Salmonella enterica serovars anatum and anatum var. 15+, isolated from retail ground turkey. Genome Announc. 4:e01619-15. doi:10.1128/genomeA.01619-15

McGhie, E. J., Brawn, L. C., Hume, P. J., Humphreys, D., and Koronakis, V. (2009). Salmonella takes control: effector-driven manipulation of the host. Curr. Opin. Microbiol. 12, 117-124. doi: 10.1016/j.mib.2008.12.001

McWhorter, A. R., and Chousalkar, K. K. (2015). Comparative phenotypic and genotypic virulence of Salmonella strains isolated from Australian layer farms. Food Microbiol. 6:12. doi: 10.3389/fmicb.2015.00012

Morgan, E., Bowen, A. J., Carnell, S. C., Wallis, T. S., and Stevens, M. P. (2007). SiiE is secreted by the Salmonella enterica Serovar typhimurium pathogenicity island 4-encoded secretion system and contributes to intestinal colonization in cattle. Infect. Immun. 75, 1524-1533. doi: 10.1128/IAI.01438-06

Mullane, N. R., Iversen, C., Healy, B., Walsh, C., Whyte, P., Wall, P. G., et al. (2007). Enterobacter sakazakii an emerging bacterial pathogen with implications for infant health. Minerva Pediatr. 59, 137-148.

Needleman, S. B., and Wunsch, C. D. (1970). A general method applicable to the search for similarities in the amino acid sequence of two proteins. J. Mol. Biol. 48, 443-453. doi: 10.1016/0022-2836(70)90057-4

Page, A. J., Cummins, C. A., Hunt, M., Wong, V. K., Reuter, S., Holden, M. T. G., et al. (2015). Roary: rapid large-scale prokaryote pan genome analysis. Bioinformatics 31, 3691-3693. doi: 10.1093/bioinformatics/btv421

Ribot, E. M., Fair, M. A., Gautom, R., Cameron, D. N., Hunter, S. B., Swaminathan, B., et al. (2006). Standardization of pulsed-field gel electrophoresis protocols for the subtyping of Escherichia coli O157: H7, Salmonella, and Shigella for PulseNet. Foodbourne Pathog. Dis. 3, 59-67. doi: 10.1089/fpd.2006.3.59

Rowe, B., Begg, N. T., Hutchinson, D. N., Dawkins, H. C., Gilbert, R. J., Jacob, M., et al. (1987). Salmonella ealing infections associated with consumption of infant dried milk. Lancet 2, 900-903. doi: 10.1016/S0140-6736(87) 91384-5
Ruby, T., McLaughlin, L., Gopinath, S., and Monack, D. (2012). Salmonella's long-term relationship with its host. FEMS Microbiol. Rev. 36, 600-615. doi: 10.1111/j.1574-6976.2012.00332.x

Rychlik, I., Karasova, D., Sebkova, A., Volf, J., Sisak, F., Havlickova, H., et al. (2009). Virulence potential of five major pathogenicity islands (SPI-1 to SPI-5) of Salmonella enterica serovar Enteritidis for chickens. BMC Microbiol. 9:268. doi: 10.1186/1471-2180-9-268

Seemann, T. (2014). Prokka: rapid prokaryotic genome annotation. Bioinformatics 30, 2068-2069. doi: 10.1093/bioinformatics/btu153

Threlfall, E. J., Ward, L. R., Hampton, M. D., Ridley, A. M., Rowe, B., Roberts, D., et al. (1998). Molecular fingerprinting defines a strain of Salmonella enterica serotype Anatum responsible for an international outbreak associated with formula-dried milk. Epidemiol. Infect. 121, 289-293. doi: 10.1017/ S0950268898001149

Wood, M. W., Jones, M. A., Watson, P. R., Hedges, S., Wallis, T. S., and Galyov, E. E. (1998). Identification of a pathogenicity island required for Salmonella enteropathogenicity. Mol. Microbiol. 29, 883-891. doi: 10.1046/j.13652958.1998.00984.x

Zheng, J., Keys, C. E., Zhao, S., Meng, J., and Brown, E. W. (2007). Enhanced subtyping scheme for Salmonella enteritidis. Emer. Infect. Dis. 13:1932. doi: 10.3201/eid1312.070185

Conflict of Interest Statement: The authors declare that the research was conducted in the absence of any commercial or financial relationships that could be construed as a potential conflict of interest.

Copyright (๑) 2016 Gunn, Finn, Hurley, Bai, Wall, Iversen, Threlfall and Fanning. This is an open-access article distributed under the terms of the Creative Commons Attribution License (CC BY). The use, distribution or reproduction in other forums is permitted, provided the original author(s) or licensor are credited and that the original publication in this journal is cited, in accordance with accepted academic practice. No use, distribution or reproduction is permitted which does not comply with these terms. 